\title{
Role of TRPV1 channels during the acquisition of fertilizing ability in boar spermatozoa
}

\author{
N. Bernabò • M. G. Pistilli • G. Falasca • V. Curini • \\ M. L. A. Garofalo • M. Turriani • M. Mattioli • \\ B. Barboni
}

Published online: 2 May 2010

(C) Springer Science+Business Media B.V. 2010

\begin{abstract}
Recently, the transient receptor potential vanilloid type 1 (TRPV1) channel was shown to be involved in capacitation, the process that allows mammalian spermatozoa to acquire their fertilizing ability within the female genital tract. Unfortunately, the role of TRPV1 in this process is still unclear. Thus, the aims of the present work were to 1) investigate the function of TRPV1 in the male gamete signaling system and 2) modulate TRPV1 activity by administering a specific activator, capsaicin, or a specific inhibitor, capsazepin, to spermatozoa during in vitro capacitation. Using confocal microscopy, cellular responses were assessed in terms of changes in 1) cell membrane resting potential, 2) intracellular calcium concentrations, and 3) actin polymerization dynamics. As a result, TRPV1 channels were shown to act as specific cationic channels: their activation led to membrane depolarization and, consequently, the opening of voltage-gated calcium channels and an increase in intracellular calcium concentrations. These ionic events promote actin cytoskeletal depolymerization and a loss of acrosome structure integrity. In contrast, TRPV1 inhibition caused a slowing of the capacitation-dependent increase in intracellular calcium concentrations, a reduction in actin polymerization, and acrosome rupture. In conclusion, these results suggest that TRPV1 channels modulate the major pathways involved in capacitation.
\end{abstract}

Keywords Capacitation $\cdot$ Acrosome reaction $\cdot$ TRPV1 receptor $\cdot$ Swine
Abbreviations
AR acrosome reaction
CB1R cannabinoid receptors type 1
CPS capsaicine

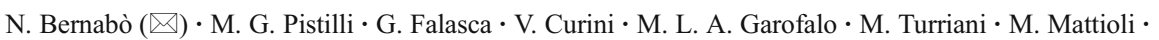
B. Barboni Department of Comparative Biomedical Sciences, University of Teramo, Piazza A. Moro 45, 64100 Teramo, Italy e-mail: nbernabo@unite.it
} 
CPZ capsazepin

OAS outer acrosome membrane

TRPV1 vanilloid receptor type 1

VGCC voltage gated calcium channels

\section{Introduction}

Immediately after ejaculation, the spermatozoa of eutherian organisms are unable to fertilize oocytes. They reach full fertilizing competence only after the completion of a complex maturation process, capacitation, within the female genital tract. Capacitation requires a few hours to days depending on the species, and allows for the presence of fertilizing spermatozoa prior to ovulation, the timing of which is not a priori. Since capacitation plays a key role in reproduction, it is necessary to coordinate capacitation with ovarian activity, because ovarian activity controls the presence of oocytes in the fertilization site. Thus, a functional dialogue exists between male and female cells in the oviduct, which acts as an interface between the two systems.

At the cellular level several biochemical factors are able to activate or inhibit the kinetics of capacitation. The calcium and bicarbonate concentrations, increased temperature, interactions with tubaric fluid proteins, and availability of energetic substrates exert capacitation-stimulatory activities (Hunter 2008). All these factors are under the control of the female endocrine axis and determine several modifications in the spermatozoa biochemistry involving all the cellular components (Salicioni et al. 2007). The concentration of second messengers, such as $\mathrm{Ca}_{2}{ }^{+}$and cAMP, rise within the cytoplasm and protein phosphorylation increases. In addition, actin polymerization forms a network between the plasma membrane (PM) and the outer acrosome membrane (OAM). The cell membrane hyperpolarizes and the lipid disorder increases, thus breaking the membrane asymmetry and exposing the cholesterol. Successively, this sterol is complexed and extracted by extracellular proteins, leading to the increase of unsaturated fatty acid-relative concentrations and membrane instability. As a consequence, the PM and OAM tend to fuse, but the fusion is blocked by the actin network interposition. All these events make the spermatozoa able to recognize and respond to the zona pellucida binding with the acrosome exocytosis (acrosome reaction, AR). The AR allows the sperm cell to penetrate through the zona pellucida and to fuse with the oocyte.

Recently, the endocannabinoid system has been described as a capacitation-inhibitory factor. The endocannabinoid system is a family of bioactive lipids synthesized on-demand from membrane acrachidonic acid that interact with many receptors. In particular, in swine spermatozoa the cannabinoid receptors type 1 (CB1R) and the vanilloid receptor type 1 (TRPV1) have been identified. CB1R is coupled to G-inhibitory proteins and act to reduce cAMP concentrations thereby slowing the first phases of fertilizing ability acquisition. On the contrary the data on the TRPV1 receptors are still unclear. TRPV1 receptors are $95-\mathrm{kDa}$ proteins constituted by six subunits (S1-S6). S5 and S6 define a pore and the C- and Ntermini are cytosolic and interact with several signal transduction systems. These molecules are non-selective cationic channels $(\mathrm{PNa} / \mathrm{PNa} \sim 10)$ and are defined as "heat/proton/lipid/ voltage-modulated $\mathrm{Ca}^{2+}$-permeant ion channels" (Ferrer-Montiel et al. 2004). They are activated by voltage, heat (temperature $>43^{\circ} \mathrm{C}$ ), and vanilloid molecules, such as capsaicine (CPS) and resinferatoxin, while they are inactivated by capsazepin (CPZ).

Initially, TRPV1s were identified in the central and peripheral nervous system where they are involved in nociception, termoception, hearing, and satiety. Successively these receptors were found in keratinocytes, epatocytes, polymorpho-nuclear granulocytes, 
macrophages, and spermatozoa (Maccarrone et al. 2005). The aim of this work was to investigate the effects exerted on spermatozoa by TRPV1 during the acquisition of fertilizing ability by use of specific agonists (CPS) and antagonists (CPZ). In particular, the effects on resting membrane potentials, intracellular $\mathrm{Ca}^{2+}$ concentrations, and actin polymerization were studied.

\section{Materials and methods}

The semen from three boars of proven fertility was collected, transferred to the laboratory, and washed from seminal plasma by a Percoll discontinuous gradient (35-70\%). The cell pellet was washed in Dulbecco with $\mathrm{Ca}^{2+}, \mathrm{Mg}^{2+}$, and $0.4 \%$ BSA and resuspended in a capacitating buffer (TCM199 with $2.25 \mathrm{mM} \mathrm{Ca}^{2+}$ lactate, $1.25 \mathrm{mM} \mathrm{Na}^{+}$pyruvate, and $13.9 \mathrm{mM}$ glucose). The cells were incubated at $38.5^{\circ} \mathrm{C}$ with $5 \% \mathrm{CO}_{2}$ in a humidified atmosphere for $4 \mathrm{~h}$ at a final concentration of $1 \times 10^{8}$ cells $/ \mathrm{ml}$. The kinetics of fertilizing ability acquisition was measured by verifying the percentage of spermatozoa undergoing AR after the incubation with solubilised zone pellucide (sZP). To this aim the amount of sperm cells losing their acrosome integrity was assessed before and after the sZP addition using a FITC-conjugated lectin (FTIC-PSA) that bound the acrosome content. The difference between the two values was considered to correspond to the percentage of capacitated spermatozoa.

The sperm cells were incubated in the presence of $1 \mathrm{mM} \mathrm{CPS}$ or $1 \mathrm{mM} \mathrm{CPZ}$ and voltage gated calcium channels (VGCC) or $\mathrm{Na}^{+}$channel-specific blocking agents (1 mM verapamil and $1 \mathrm{mM} \mathrm{NiCl}$, or $10 \mu \mathrm{M}$ tetrodotoxin, respectively) were used. Membrane depolarization and intracellular calcium concentrations $\left(\left[\mathrm{Ca}^{2+}\right]_{\mathrm{i}}\right)$ were assessed using the fluorescent stains bis-oxonol and Fluo-3-AM. Actin polymerization was evaluated by confocal microscopy using the FITC-conjugated falloidin, a fluorescent molecule able to selectively bind F-actin. The intracellular distribution of polymeric actin (F-actin) was assessed by the LaserPix 4.0 (BioRad) software for single cell reconstruction of the 3D signal. The data are expressed as mean \pm standard deviation of at least three independent determinations.

\section{Results}

The 4-h incubation of spermatozoa promoted capacitation achievement in $30-40 \%$ of the cells. Moreover, at the end of the incubation, the sperm treatment with TRPV1 modulators resulted in a significant increase in the spontaneous AR percentages (CTR $12.5 \pm 1.2 \%$ vs. CPS $45.2 \pm 5.6 \%$ and CPZ $38.5 \pm 4.4 \%$; $<<0.001$ by ANOVA). The functional analyses demonstrated that the CPS caused membrane depolarization visible as an increase in bisoxonol fluorescence emission independent of the completion of capacitation in about $60 \%$ of the cells. In addition, the CPS determined a rapid, 60 long $\left[\mathrm{Ca}^{2+}\right]_{\mathrm{i}}$ surge, in more than $50 \%$ of the spermatozoa (Fluo-3-AM). The contemporaneous presence of VGCC blocking agents (verapamil or $\mathrm{NiCl}$ ) or the absence of extracellular $\mathrm{Ca}^{2+}$ prevented the CPS effect. On the contrary, these treatments did not alter the CPS-induced modification of the membrane resting potential.

The addition of CPZ to the observation chamber did not alter the membrane resting potential while it promoted $\left[\mathrm{Ca}^{2+}\right]_{\mathrm{i}}$ lowering. Finally, during the incubation in capacitating conditions, the actin specifically polymerized in the sperm acrosomal region. The CPS treatment caused a rapid actin depolymerization at the acrosome level in more than $40 \%$ of 
the spermatozoa, thus determining the loss of acrosome integrity. The CPZ during the $4 \mathrm{~h}$ incubation caused a decrease in normal actin polymerization and the consequential loss of acrosome integrity in more than $50 \%$ of the cells.

\section{Discussion}

These results defined the role of TRPV1 channels during the acquisition of fertilizing ability in boar spermatozoa. Together with their function as non-selective cationic channels, these molecules demonstrated a control mechanism of membrane potential and ionic fluxes. In particular, TRPV1 activation promoted a $\mathrm{Na}^{+}$influx and, as a consequence, a depolarization wave. This event caused the activation of VGCC and an increase in $\left[\mathrm{Ca}^{2+}\right]_{\mathrm{i}}$. The alterations of calcium concentration were responsible for the actin polymerization status and the integrity of the acrosome structure.

In conclusion, the TRPV1 channels are involved in intracellular signal transduction during the acquisition of fertilizing ability and the AR. These data enhance the basic knowledge and improve applicative strategies in male reproductive physiopathology.

\section{References}

Ferrer-Montiel A, García-Martínez C, Morenilla-Palao C, García-Sanz N, Fernández-Carvajal A, FernándezBallester G, Planells-Cases R (2004) Molecular architecture of the vanilloid receptor. Insights for drug design. Euro J Biochem 271:1820-1816

Hunter RH (2008) Sperm release from oviduct epithelial binding is controlled hormonally by per-ovulatory graafian follicles. Mol Reprod Develop, 75:167-174

Maccarrone M, Barboni B, Paradisi A, Bernabò N, Gasperi V, Pistilli MG, Fezza F, Lucidi P, Mattioli M (2005) Characterization of the endocannabinoid system in boar spermatozoa and implications for sperm capacitation and acrsome reaction. J Cell Sci 118:4393-4404

Salicioni AM, Platt MD, Wertheimer EV, Arcelay E, Allaire A, Sosnik J, Visconti PE (2007) Signalling pathways involved in sperm capacitation. Soc Reprod Fertil Suppl 65:245-259 